\title{
Single-level anterior cervical discectomy and fusion versus minimally invasive posterior cervical foraminotomy for patients with cervical radiculopathy: a cost analysis
}

\author{
Haley E. Mansfield, M.S., ${ }^{1}$ W. Jeffrey Canar, Ph.D., ${ }^{2}$ Carter S. Gerard, M.D., ${ }^{1}$ \\ AND John E. O'ToOLE, M.D., M.S. ${ }^{1}$ \\ Departments of ${ }^{1}$ Neurological Surgery and ${ }^{2}$ Health Systems Management, Rush University, Chicago, Illinois
}

\begin{abstract}
Object. Patients suffering from cervical radiculopathy in whom a course of nonoperative treatment has failed are often candidates for a single-level anterior cervical discectomy and fusion (ACDF) or posterior cervical foraminotomy (PCF). The objective of this analysis was to identify any significant cost differences between these surgical methods by comparing direct costs to the hospital. Furthermore, patient-specific characteristics were also considered for their effect on component costs.

Methods. After obtaining approval from the medical center institutional review board, the authors conducted a retrospective cross-sectional comparative cohort study, with a sample of 101 patients diagnosed with cervical radiculopathy and who underwent an initial single-level ACDF or minimally invasive PCF during a 3-year period. Using these data, bivariate analyses were conducted to determine significant differences in direct total procedure and component costs between surgical techniques. Factorial ANOVAs were also conducted to determine any relationship between patient sex and smoking status to the component costs per surgery.

Results. The mean total direct cost for an ACDF was $\$ 8192$, and the mean total direct cost for a PCF was $\$ 4320$. There were significant differences in the cost components for direct costs and operating room supply costs. It was found that there was no statistically significant difference in component costs with regard to patient sex or smoking status.

Conclusions. In the management of single-level cervical radiculopathy, the present analysis has revealed that the average cost of an ACDF is $89 \%$ more than a PCF. This increased cost is largely due to the cost of surgical implants. These results do not appear to be dependent on patient sex or smoking status. When combined with results from previous studies highlighting the comparable patient outcomes for either procedure, the authors' findings suggest that from a health care economics standpoint, physicians should consider a minimally invasive PCF in the treatment of cervical radiculopathy.

(http://thejns.org/doi/abs/10.3171/2014.8.FOCUS14373)
\end{abstract}

\begin{abstract}
KEY WORDS - cervical radiculopathy - anterior cervical discectomy and fusion • minimally invasive posterior cervical foraminotomy
\end{abstract}

$\mathrm{C}$

ERVICAL radiculopathy is one of the most common problems addressed by spinal surgeons. AccordQuality, in 2009 approximately 32,234 patients were discharged with ICD-9 codes related to neck pain. Of those $18.7 \%(\mathrm{n}=6020)$ were patients diagnosed with cervical radiculopathy.

There are various nonoperative treatment options for cervical radiculopathy including physical therapy, medical management, and epidural steroid injections. Nonsurgical treatment options have been shown to have up to a $90 \%$ success rate. ${ }^{8}$ For those patients in whom nonoperative treatment fails, surgery is a viable alternative. Two of the most common procedures performed include anterior cer-

\footnotetext{
Abbreviations used in this paper: $\mathrm{ACDF}=$ anterior cervical discectomy and fusion; BMI = body mass index; LOS = length of stay; $\mathrm{PCF}=$ posterior cervical foraminotomy.
}

vical discectomy and fusion (ACDF) and posterior cervical foraminotomy (PCF). The surgical intervention offered to patients is at the discretion of the surgeon, and not all surgeons perform an ACDF or PCF interchangeably for patients with cervical radiculopathy. An ACDF requires removing the entire disc and associated osteophytes and implanting a bone graft typically with instrumentation to fuse the vertebrae adjacent to the disc. A PCF requires creating a small bony opening over the affected nerve root and often removing a specific fragment of herniated disc compressing the nerve, but no bone graft or instrumentation is required. Additionally, a PCF can be done with a minimally invasive approach.

There is controversy regarding the best surgical intervention for patients with cervical radiculopathy, considering both patient outcomes and procedure costs. Regarding patient outcomes, studies have shown that both ACDF and PCF produce similar quality-adjusted life 
years and similar clinical benefit. . $^{1,5,6,10,13}$ Because there is no fusion, PCF should allow for greater retention of neck mobility. The procedure is also somewhat less invasive than ACDF; however, study findings have reported that in up to $3.3 \%$ of cases, postoperative symptoms may recur, necessitating subsequent surgery typically with fusion. ${ }^{4}$ On the other hand, ACDF reduces mobility of the neck at the surgical level and exposes the patient to risks associated with the anterior neck approach, including dysphagia, dysphonia, and large vessel or pharyngeal injury. ${ }^{9}$ From a financial perspective, a prior study reported the average total direct cost (specifically institution and instrumentation related costs) equaled $\$ 10,078$ per ACDF and $\$ 3570$ for a $\mathrm{PCF} .{ }^{14} \mathrm{An} \mathrm{ACDF}$ incurs higher operating room costs because of the time spent on instrumentation implantation. ${ }^{2,3}$ Because a PCF does not involve implantation and is minimally invasive, it logically incurs less operating room time and cost. Importantly, additional costs beyond operating room costs that make up the total for each procedure have not been investigated.

The objective of this analysis was to identify all significant cost differences between these surgical procedures through a comparison of direct costs to the hospital including both total procedure and procedure component costs. Only direct costs were considered in the present study, advancing the findings of previous studies by breaking down total cost per procedure into total; operating room supplies; anesthesia; time in the operating room, recovery room, observation room, and inpatient room; imaging; pharmacy; and inpatient physical and occupational therapy. Furthermore, specific patient demographic characteristics, including sex and smoking status, were included as covariates.

\section{Methods}

Subsequent to obtaining approval from the Medical Center Institutional Review Board, a retrospective crosssectional comparative cohort study was performed for 101 patients diagnosed with cervical radiculopathy who underwent an initial single-level ACDF $(n=79)$ or minimally invasive PCF $(n=22)$. Patients were considered eligible for the study if they had symptomatic single-level cervical degenerative disease presenting with radiculopathy. Exclusion criteria were 1) symptomatic cervical myelopathy, 2) prior spine surgery at any level, and 3) evidence of cervical instability.

\section{Patient Selection}

All surgeries were performed at a single institution, in the main operating room, by 3 surgeons during the period 2009-2012. All patients were considered candidates for either procedure given the unilateral and noncentral nature of their disc herniation or stenosis. Selection of the procedure type was based on surgeon discretion predominantly due to nonquantifiable parameters, including surgeon preference or undefined patient characteristics. In general, $\mathrm{PCF}$ patients were scheduled for surgery the same day as surgery discharge, whereas ACDF patients were scheduled for 23-hour observation status, although adherence to these schedules was not universal. Although scheduled for same-day discharge or 23-hour observation status, some patients were admitted $(n=25)$, with 23 of 25 ACDF patients (92\%) admitted beyond 23 hours. Eligibility for this study was determined through a manual chart review. Once the sample was identified, a complete review of medical records was conducted to gather patient age; sex; body mass index (BMI); length of stay (LOS); smoking status; marital status; employment status; alcohol use; presence of osteoporosis, diabetes, and rheumatoid arthritis; disability status; and postoperative hospital encounters, admissions, and surgeries. However, due to the low presence of osteoporosis, diabetes, and rheumatoid arthritis; disability status; and postoperative hospital encounters, admissions, and surgeries across both surgery groups, the final analysis only included patient age, sex, marital status, smoking history, employment status, history of alcohol use, BMI, LOS, and primary payer (Table 1). Factors such as payer, marital status, employment status, and history of alcohol use were chosen because they may be surrogate markers for different socioeconomic groups and may have an impact on LOS, medication usage, and other related costs that can impact the overall direct cost to the hospital.

\section{Breakdown of Cost}

Our institutional Medical Center Decision Support team provided all cost data associated with the surgical encounter from admission to discharge. The cost data represent the direct cost incurred to the hospital per patient encounter, not including physician fees or indirect costs. These costs included operating room supplies (including instrumentation); anesthesia (excluding physician fees); time in operating room (including preoperative time) recovery room, observation room, and inpatient room; imaging, pharmacy, and inpatient occupational and physical therapy. The total cost for the procedure was defined as the sum of direct costs associated with each surgical encounter from admission to discharge. Instrumentation cost was unable to be extracted from the data set and therefore those costs were bundled into the operating room supplies cost component. Operating room time was defined as the time the operating room was used, including the time the patient spent in the preoperative holding area. Preoperative utilization for each procedure and direct cost analysis data are described in Tables 2 and 3.

All statistical analysis was performed using PASW Statistics for Windows, (version 18.0, SPSS Inc.). Chisquare tests were conducted to determine the association between control variables and LOS and surgery type. Bivariate t-tests were performed to test the difference between surgery type and total average cost. Due to the number of categorical variables in the study, factorial ANOVAs were performed to determine the costs associated with the surgery type, accounting for patient sex and smoking status.

\section{Results}

Three ACDF patients and 1 PCF patient had an average direct cost of $\$ 36,613$. Because of this aberrant variance and to allow for more practical comparisons, these 


\section{Cost analysis of ACDF vs foraminotomy}

TABLE 1: Demographics of patients for each procedure $(n=97)^{*}$

\begin{tabular}{|c|c|c|c|}
\hline Variable & ACDF Group $(n=76)$ & PCF Group $(n=21)$ & $p$ Value \\
\hline age in yrs & & & 0.952 \\
\hline mean & 49 & 49 & \\
\hline range & $24-75$ & $31-69$ & \\
\hline mean BMI $\left(\mathrm{kg} / \mathrm{m}^{2}\right)$ & $28 \pm 5.8$ & $29 \pm 5.9$ & 0.282 \\
\hline smoker & $18(23.7)$ & $5(23.8)$ & 0.990 \\
\hline osteoporosis & $2(2.6)$ & $0(0.0)$ & 0.453 \\
\hline diabetes & $10(13.2)$ & $1(4.8)$ & 0.447 \\
\hline rheumatoid arthritis & $3(3.9)$ & $0(0.0)$ & 0.353 \\
\hline disability & $6(7.89)$ & $1(4.8)$ & 0.644 \\
\hline patient sex & & & 0.368 \\
\hline male & 35 (46.1) & $12(57.1)$ & \\
\hline female & $41(53.9)$ & $9(42.9)$ & \\
\hline payer & & & 0.216 \\
\hline private & $51(67.1)$ & $13(61.9)$ & \\
\hline government & $22(28.9)$ & $5(23.8)$ & \\
\hline other & $3(3.9)$ & $3(14.3)$ & \\
\hline marital status $\dagger$ & & & 0.069 \\
\hline divorced & $6(9.2)$ & $1(5.2)$ & \\
\hline married & $45(69.2)$ & $10(52.6)$ & \\
\hline single & $14(21.5)$ & $6(31.6)$ & \\
\hline widowed & $0(00.0)$ & $2(10.5)$ & \\
\hline employment status $\ddagger$ & & & 0.766 \\
\hline employed & $35(62.5)$ & $10(66.7)$ & \\
\hline not employed & $21(37.5)$ & $5(33.3)$ & \\
\hline
\end{tabular}

4 patients were excluded from the subsequent analysis due to excessive operating room expenses related to extra screw implantation and mechanical ventilation. The final analytical sample included 97 patients: 76 ACDF patients and 21 PCF patients.

We investigated the variables age; BMI; patient sex; LOS; smoking, marital, and employment status; and payer to determine if there was an association with surgery type. The data revealed a significant association between LOS and surgery type $\left(\mathrm{t}_{95}=3.202, \mathrm{p}=0.002\right)$. The average LOS for PCF was $0.57 \pm 0.87$ days and ACDF $1.41 \pm$

TABLE 2: Preoperative utilization for each procedure $(n=97)^{*}$

\begin{tabular}{lccc}
\hline \multicolumn{1}{c}{ Variable } & $\begin{array}{c}\text { ACDF Group } \\
(n=76)\end{array}$ & $\begin{array}{c}\text { PCF Group } \\
(n=21)\end{array}$ & p Value \\
\hline LOS (days) & $1.41 \pm 1.21$ & $0.57 \pm 0.87$ & 0.002 \\
postoperative reop & $2(2.63)$ & $0(00.0)$ & \\
readmitted to hospital & $0(0.00)$ & $0(00.0)$ & \\
postoperative hospital & $2(2.63)$ & $0(00.0)$ & \\
encounter & & & \\
\hline * Values are number of patients (\%) unless noted otherwise. The LOS &
\end{tabular}

Neurosurg Focus / Volume 37 / November 2014
1.21 days; however, there was no significant association between surgery type and the variables age; BMI; patient sex; smoking, marital, and employment status; and payer. In the ACDF group, 2 patients underwent reoperation; one patient underwent a single-level PCF at the index level 1 year postoperatively and the other patient underwent a lumbar laminectomy 6 months postoperatively. Two patients had a postoperative hospital encounter following their initial procedure; one patient presented to the emergency department with shortness of breath and the other patient presented to the emergency department for pain. No patients were readmitted to the hospital postoperatively. No patients in the PCF group underwent reoperation, had a postoperative hospital encounter, or underwent readmission at the study institution. These results are illustrated in Table 2.

Cost data were collected to determine cost components that make up the total direct cost of each procedure. Table 3 summarizes the bivariate statistical relationship between surgery type and cost components, and the overall main effect for surgery type and cost after controlling for patient sex and smoking status. PCF patients incurred an average total direct cost of $\$ 4320 \pm \$ 1719$, including operating room supplies that averaged \$1304. In comparison, patients who underwent an ACDF incurred an 
H. E. Mansfield et al.

TABLE 3: Direct cost analysis and main effect of surgery type $(n=97)$

\begin{tabular}{lrrrrr}
\hline \multicolumn{1}{c}{ Cost Component } & ACDF $^{*}$ & PCF $^{*}$ & Difference $^{*}$ & $F$ & $p$ Value \\
\hline total direct cost per encounter & $\$ 8192$ & $\$ 4320$ & $\$ 3872$ & 4.703 & $<0.001$ \\
operating room supplies & $\$ 4447$ & $\$ 1304$ & $\$ 3143$ & 5.032 & $<0.001$ \\
anesthesia & $\$ 421$ & $\$ 405$ & $\$ 16$ & 0.584 & 0.767 \\
time in operating room & $\$ 1534$ & $\$ 1363$ & $\$ 171$ & 0.841 & 0.556 \\
time in recovery room & $\$ 285$ & $\$ 454$ & $(\$ 169)$ & 1.991 & 0.065 \\
time in observation room & $\$ 592$ & $\$ 615$ & $(\$ 23)$ & & 0.475 \\
time in inpatient room & $\$ 913$ & $\$ 660$ & $\$ 253$ & 0.948 & 0.568 \\
imaging & $\$ 160$ & $\$ 129$ & $\$ 31$ & 0.826 & 0.484 \\
pharmacy & $\$ 306$ & $\$ 240$ & $\$ 66$ & 0.935 & 0.676 \\
inpatient therapy & $\$ 114$ & $\$ 78$ & $\$ 36$ & 0.694 & \\
\hline * Mean values. & & & & &
\end{tabular}

average total direct cost of $\$ 8192 \pm \$ 3155$, with operating room supplies, including instrumentation, averaging $\$ 4447$. This resulted in an average difference of $\$ 3872$ between the 2 procedures and an average difference in cost of operating room supplies of \$3143. Additional follow-up analyses indicated significant differences in costs per surgery type for operating room supply cost. These results are illustrated in Table 3 .

The overall main effect was significant for surgery type and cost, after controlling for patient sex and smoking status that was evident for total cost $(\mathrm{F}=4.703, \mathrm{p}<$ $0.001)$ and operating room supply cost $(F=5.032, p<$ 0.001). However, there was no significant main effect for anesthesia $(\mathrm{F}=0.584, \mathrm{p}=0.767)$; time in the operating room $(\mathrm{F}=0.841, \mathrm{p}=0.556)$, recovery room $(\mathrm{F}=1.991$, $\mathrm{p}=0.065)$, observation room, and inpatient room $(\mathrm{F}=$ $0.948, \mathrm{p}=0.475)$; imaging $(\mathrm{F}=0.826, \mathrm{p}=0.568)$; pharmacy $(\mathrm{F}=0.935, \mathrm{p}=0.484)$; or therapy costs $(\mathrm{F}=0.694$, $\mathrm{p}=0.676)$. Additionally, there was no significant association between surgery type and LOS after controlling for patient sex and smoking status $(\mathrm{F}=1.634, \mathrm{p}=0.136)$.

\section{Discussion}

Ideal clinical care provides treatments that produce good patient outcomes but that are also cost conscious. To this end, the present work provides some insight in assessing the cost of 2 common spinal surgical procedures. As expected, our study findings prove that there were significant differences in total direct cost per encounter, with ACDF costing 89\% more than PCF. These results are in agreement with previous findings. ${ }^{1-3,6,12}$ The present work, however, considers a more comprehensive list of direct cost components to the hospital. Of the 10 cost components studied in our sample of 97 patients, an ACDF cost more than a PCF in 8 of the 10 categories studied, including total direct costs; operating room supplies; anesthesia; time in the operating room and inpatient room; imaging; pharmacy; and inpatient therapy. Cost differences in anesthesia; time in the operating room, recovery room, observation room, and inpatient room, imaging; pharmacy; and inpatient therapy were not significant when controlling for sex and smoking status. These findings also support the notion that operating room supplies, including instrumentation, contribute to the majority of the cost difference between ACDF and PCF.

\section{Study Limitations}

Due to our limited sample size, it was not possible to investigate the effect of significant comorbidities such as diabetes, rheumatoid arthritis, osteoporosis, and disability on costs. A second limitation to this study is that it does not account for patients who did not return to the institution for retreatment care (e.g., complication or reoperation). Additionally, this data set allows only a cost analysis and not a cost-effectiveness comparison due to the absence of patient-reported clinical outcomes. However, the lack of differences in 90-day and 2-year retreatment events may act as a rough surrogate for similar overall patient outcomes. Furthermore, this study cannot account for unique clinical patient-specific factors that drive surgical decision making independent of cost, such as subtle differences in pathology, patient preference, or surgeon bias. Therefore, there is an obvious need for further prospective comparative cost-effectiveness analyses to better establish rational treatment decision making based on patient outcomes and cost.

Overall, the cost differences we found were anticipated and keep within the findings of previously reported studies that looked at cost, as well as clinical outcomes comparing these 2 procedures., ${ }^{2,11}$ In carefully selected patients, the literature has shown that decompression for cervical radiculopathy can be accomplished with equal efficacy with either a PCF or an ACDF. ${ }^{5-7,11}$ The major cost difference per procedure demonstrated here might prompt surgeons to more actively weigh cost considerations in technique selection when confronted with patients eligible for either procedure. By identifying the major sources of cost as operating room supplies, including instrumentation, physicians may be more able to make focused changes in practice to significantly reduce the charges incurred by the patient. The costs incurred per procedure not only affect the patient and payers but also the hospital. From a hospital economics standpoint, if more patients are discharged on the same day as their surgery (as with PCF), the hospital theoretically has a 
greater number of inpatient beds available to treat other cost-incurring patients, thereby directly influencing local and regional patient-care volumes.

As mentioned above, our study is limited by its retrospective design and the lack of accompanying clinical follow-up data. Additionally, this paper is hindered in that it is unable to provide key costs that may influence decision making such as physician fees and the exact cost of instrumentation. Establishing a definitive indication for PCF versus single-level ACDF in isolated cervical radiculopathy is beyond the scope of this paper. Yet, when equipoise exists between the procedures for an individual case, cost data for each approach may influence the surgeon in his or her clinical decision-making process. While this article attempts to address the lack of transparency in regard to the cost of spinal surgical care, there remains a substantial need for further prospective comparative cost-effectiveness analyses to better establish rational treatment decision making based on patient outcomes and cost.

\section{Conclusions}

Surgical decision making regarding the choice of ACDF versus PCF procedures for the treatment of cervical radiculopathy remains unclear. A detailed statistical analysis of patient outcome and cost data for a sample of 97 patients has revealed that in the management of singlelevel cervical radiculopathy the cost of an ACDF is $89 \%$ more than a PCF with the greatest disparity caused by operating room supplies, including instrumentation. When combined with results from previous studies highlighting the comparable patient outcomes for either procedure, this study may serve to influence the choice of surgical technique and allows for focused modifications in clinical practice to reduce cost and ensure greater utility of the shrinking health care dollar.

\section{Acknowledgment}

We thank Lee A. Tan, M.D., for his assistance with editing.

\section{Disclosure}

The manuscript has been approved by all of the authors. No funding was received for this study. Dr. O'Toole is a consultant for Globus Medical, Pioneer Surgical, RTI Surgical, Nexxt Spine, and the Food and Drug Administration, and receives royalties from Globus Medical and Pioneer Surgical. The manuscript has been prepare in accordance with the institutional review board at the authors' institution and is in accordance with accepted ethical standards.

Author contributions to the study and manuscript preparation include the following. Conception and design: Mansfield, O'Toole. Acquisition of data: Mansfield. Analysis and interpretation of data: Mansfield, Canar. Drafting the article: Mansfield. Critically revising the article: all authors. Reviewed submitted version of manuscript: all authors. Statistical analysis: Mansfield, Canar. Study supervision: Canar, O’Toole.

\section{References}

1. Alvin MD, Lubelski D, Abdullah KG, Whitmore RG, Benzel EC, Mroz TE: Cost-utility analysis of anterior cervical discec- tomy and fusion with plating (ACDFP) versus posterior cervical foraminotomy (PCF) for patients with single-level cervical radiculopathy at 1-year follow-up. J Spinal Disord Tech [epub ahead of print], 2014

2. Angevine PD, Zivin JG, McCormick PC: Cost-effectiveness of single-level anterior cervical discectomy and fusion for cervical spondylosis. Spine (Phila Pa 1976) 30:1989-1997, 2005

3. Carreon LY, Anderson PA, Traynelis VC, Mummaneni PV, Glassman SD: Cost-effectiveness of single-level anterior cervical discectomy and fusion five years after surgery. Spine (Phila Pa 1976) 38:471-475, 2013

4. Clarke MJ, Ecker RD, Krauss WE, McClelland RL, Dekutoski MB: Same-segment and adjacent-segment disease following posterior cervical foraminotomy. J Neurosurg Spine 6:5-9, 2007

5. Henderson CM, Hennessy RG, Shuey HM Jr, Shackelford EG: Posterior-lateral foraminotomy as an exclusive operative technique for cervical radiculopathy: a review of 846 consecutively operated cases. Neurosurgery 13:504-512, 1983

6. Herkowitz HN, Kurz LT, Overholt DP: Surgical management of cervical soft disc herniation. A comparison between the anterior and posterior approach. Spine (Phila Pa 1976) 15: 1026-1030, 1990

7. Jagannathan J, Sherman JH, Szabo T, Shaffrey CI, Jane JA: The posterior cervical foraminotomy in the treatment of cervical disc/osteophyte disease: a single-surgeon experience with a minimum of 5 years' clinical and radiographic followup. Clinical article. J Neurosurg Spine 10:347-356, 2009

8. Murphy DR, Hurwitz EL, Gregory A, Clary R: A nonsurgical approach to the management of patients with cervical radiculopathy: a prospective observational cohort study. J Manipulative Physiol Ther 29:279-287, 2006

9. Nanda A, Sharma M, Sonig A, Ambekar S, Bollam P: Surgical complications of anterior cervical diskectomy and fusion for cervical degenerative disk disease: a single surgeon's experience of 1576 patients. World Neurosurg [epub ahead of print], 2013

10. Onimus M, Destrumelle N, Gangloff S: [Surgical treatment of cervical disk displacement. Anterior or posterior approach?] Rev Chir Orthopédique Réparatrice Appar Mot 81:296301, $1995(\mathrm{Fr})$

11. Quintana LM: Complications in anterior cervical discectomy and fusion for cervical degenerative disc disease. World Neurosurg [epub ahead of print], 2013

12. Qureshi SA, McAnany S, Goz V, Koehler SM, Hecht AC: Cost-effectiveness analysis: comparing single-level cervical disc replacement and single-level anterior cervical discectomy and fusion. Clinical article. J Neurosurg Spine 19:546554,2013

13. Tomaras CR, Blacklock JB, Parker WD, Harper RL: Outpatient surgical treatment of cervical radiculopathy. J Neurosurg 87:41-43, 1997

14. Tumialán LM, Ponton RP, Gluf WM: Management of unilateral cervical radiculopathy in the military: the cost effectiveness of posterior cervical foraminotomy compared with anterior cervical discectomy and fusion. Neurosurg Focus 28(5):E17, 2010

Manuscript submitted June 24, 2014.

Accepted August 20, 2014.

Please include this information when citing this paper: DOI: 10.3171/2014.8.FOCUS14373.

Address correspondence to: Haley Mansfield, M.S., Department of Neurological Surgery, Rush University Medical Center, 1725 W. Harrison St., Professional Building Ste. 855, Chicago, IL 60612. email: haleymans@gmail.com. 